\title{
Functional insight for $\beta$-glucuronidase in Escherichia coli and Staphylococcus sp. RLH1
}

\author{
Loganathan Arul ${ }^{1, *}$, George Benita ${ }^{1}$ and Ponnusamy Balasubramanian ${ }^{1}$ \\ ${ }^{1}$ Centre for Plant Molecular Biology, Tamil Nadu Agricultural University, Coimbatore - 641 003, India; \\ Loganathan Arul* - E-mail: arulsrj@rediffmail.com; Phone: 91422 6611353; * Corresponding author \\ received March 04, 2008; revised April 24, 2008; accepted May 05, 2008; published May 22, 2008
}

\begin{abstract}
:
Glycosyl hydrolases hydrolyze the glycosidic bond either in carbohydrates or between carbohydrate and non-carbohydrate moiety. The $\beta$-glucuronidase (beta D-glucuronoside glucuronosohydrolase; EC 3.2.1.31) enzyme belongs to the family-2 glycosyl hydrolase. The E. coli borne $\beta$-glucuronidase gene (uidA) was devised as a gene fusion marker in plant genetic transformation experiments. Recent plant transformation vectors contain a novel $\beta$-glucuronidase (gusA) derived from Staphylococcus sp. RLH1 for E. coli uidA. It is known to have a ten fold higher sensitivity compared to E. coli $\beta$-glucuronidase. The functional superiority of Staphylococcus (gusA) over E. coli (uidA) activity is not fully known. The comparison of secondary structural elements among them revealed an increased percentage of random coils in Staphylococcus $\beta$-glucuronidase. The 3D model of gusA shows catalytic site residues 396Glu, 508Glu and 471Tyr of gusA in loop regions. Accessible surface area (ASA) calculations on the 3D model showed increased ASA for active site residues in Staphylococcus $\beta$-glucuronidase. Increased random coil, the presence of catalytic residues in loops, greater solvent accessibility of active residues and increased charged residues in gusA of Staphylococcus might facilitate interaction with the solvent. This hypothesizes the enhanced catalytic activity of $\beta$-glucuronidase in Staphylococcus sp. RLH1 compared to that in E. coli.
\end{abstract}

Keywords: $\beta$-glucuronidase; structure-function relationships; uidA; GUSPlus; catalytic activity

Background:

Glycosyl hydrolases are a widespread group of enzymes hydrolyzing the glycosidic bond in carbohydrates or its derivatives. $\beta$-glucuronidase (EC 3.2.1.31) is a glycosyl hydrolase which hydrolyses $\beta$-glucuronic acid residues from the non-reducing termini of glycosaminoglycans (GAGs). Protein sequences of $\beta$-glucuronidase (GUS) from prokaryotes and eukaryotes are available at NCBI GenPept. In prokaryotes, the GUS of Escherichia coli is a well investigated glycosyl hydrolase. The $E$. coli $\beta$-glucuronidase gene (uidA) has been sequenced, and it is known to encode a stable enzyme [1]. The E. coli GUS is 603 amino acid residues long and it shares about $50 \%$ sequence identity with the human GUS with same substrate specificity. The human GUS contains three structural domains, namely, the sugar binding domain, immunoglobulin like beta-sandwich domain and TIM barrel domain [2]. It is also present in E. coli GUS. Jefferson [3] developed a gene fusion system using $E$. coli GUS gene (uidA) as a marker for analyzing gene expression in plant transformation experiments. The wide use of GUS as a tool in plant gene expression was primarily due to the stability and sensitivity of the reporter assay by providing quantitative and qualitative data on transgene expression. Transgenic plants expressing GUS show histochemical staining with the substrate 5-Bromo-4-chloro-3-indolyl-betaD-glucuronide (X-Gluc) for GUS assay at $\mathrm{pH}$ 7.0.
The enzyme GUS acts by cleaving the $\beta$-glucuronic acid from the substrate X-Gluc and it produces blue color in transformed tissues. The recent version of CAMBIA plant transformation vectors (pCAMBIA 1305.1, pCAMBIA 1305.2, pCAMBIA 1105.1, pCAMBIA 1105.1R, pCAMBIA 0105.1R and pCAMBIA 0305.2) carry a novel GUS gene derived from Staphylococcus sp. RLH1. GUSPlus is a synthetic gene (gusA) from Staphylococcus.encoding GUS protein detectable at ten-fold higher sensitivity to E. coli GUS $[4,5]$. The benefits of GUSPlus over E. coli GUS are: (1) greater sensitivity; (2) fast colour development with X-Gluc substrate; and (3) higher thermal stability. However, the molecular basis for the functional difference between GUS from Staphylococcus (GUSPlus) and E. coli is not fully understood. Here, we probe their functional difference using sequence and structural data analysis.

\section{Methodology: \\ GUS sequence data:}

Amino acid sequences of family- $2 \beta$-glucuronidase (beta-Dglucuronoside glucuronosohydrolase; EC 3.2.1.31) for Homo sapiens (NP_000172), Escherichia coli K12 (AAC74689) and Staphylococcus sp. RLH1 (AAK29422) were downloaded from GenPept at NCBI [6]. 


\begin{abstract}
Sequence comparison
The percent identity shared between $\beta$-glucuronidase sequences was found by pairwise alignment using BLOSUM62 matrix [7]. The conserved catalytic site residues in $\beta$-glucuronidase were identified based on multiple sequence alignment (Figure 1) using ClustalW [8].
\end{abstract}

\section{Physical and chemical parameter estimation}

The physical and chemical parameters were predicted using the ExPASy ProtParam tool available online as described elsewhere [9]. The computed parameters include the molecular weight, theoretical pI (isoelectric point), amino acid composition, atomic composition, instability index and aliphatic index (Table 1 in supplementary material).

\section{Secondary structure analysis}

The GUS sequence from E. coli and Staphylococcus were analyzed for percentage composition of alpha helix, beta strands and random coils (Table 2) using the GORIV secondary structure prediction method available at NPS@: network protein sequence analysis [10].

\section{D model building}

3D structure of GUS from E. coli and Staphylococcus sp. were predicted using the crystal structure of human GUS as template using Discovery Studio (Accelrys Software Inc, version 1.7). The coordinates of the template human GUS (PDB Id: 1BHG) was downloaded from PDB [11]. Model validation was done using Ramachandran Plot. The resulting structure was energy minimized using the CHARMM force field and the process was repeated till the structure with desired energy levels was obtained. Superposition of the modeled structures of E. coli and Staphylococcus GUS was carried out using the Combinatorial Extension (CE) method and the root mean square deviation (RMSD) was calculated [12].

\section{Accessible surface area (ASA) calculations}

The accessible molecular surface was calculated for human, E. coli and Staphylococcus GUS model structures using WHAT IF [13]. The Statistics about accessibilities (SHOACC) option was used to find out the accessibility of residues. The side chain accessibility for the catalytic site residues of human, E. coli and Staphylococcus GUS were plotted as a function of residue position (Figure 2).

\section{Discussion:}

The functional superiority of GUS from Staphylococcus over E. coli is intriguing. However, the molecular basis of functional advantage for Staphylococcus GUS is not known. Here, we probe GUS functional dominance in Staphylococcus over E. coli using sequence analysis and predicted 3D models. Pair-wise alignment of E. coli and Staphylococcus GUS revealed an identity of $45.7 \%$ and a similarity of $63.5 \%$. Similar alignments of GUS from human and E. coli show $42.6 \%$ identity and that of human and Staphylococcus is $41.8 \%$ identity. Nevertheless, E. coli and Staphylococcus GUS showed conservation of catalytic site residues at positions corresponding to human GUS. Based on earlier reports for human $\beta$-glucuronidase, 451Glu was identified to be the proton donor and $540 \mathrm{Glu}$ as the nucleophile which stabilize the carbonium ion in the catalytic reaction [2] and [14]. 504Tyr is located in the active site of human GUS and its role in catalysis has not been defined. Results show conservation of 394Glu, 504Glu 468Tyr in E. coli and 396Glu, 508Glu, 471Tyr in Staphylococcus GUS at positions corresponding to the catalytic site residues of human GUS (Figure 1). Subsequently, the amino acid sequence of GUS from E. coli and Staphylococcus were analyzed for its physicochemical properties (Table 1 in supplementary material).

The E. coli GUS is 603 residues long with a predicted molecular weight of 68.447 KDa. Similarly, the Staphylococcus GUS is 602 amino acid residues with a molecular weight of $68.701 \mathrm{KDa}$. Further the instability index predicted for E. coli and Staphylococcus GUS did not show difference between them (instability index provides an estimate of the stability of the enzyme in a test tube). It is known that a protein whose instability index is smaller to 40 is predicted as stable, a value above 40 predicts that the protein may be unstable [15]. However, the significant functional difference observed was with respect to the composition of the charged amino acid residues. The Staphylococcus GUS contains ten negatively charged residues and six positively charged residues more than that in E. coli GUS (Table 1 see supplementary material). The increased percentage of charged residues is likely to enhance hydrophilicity in Staphylococcus GUS favoring interactions with the solvent environment. Further, analysis of the predicted secondary structures of GUS from E. coli and Staphylococcus revealed that they predominantly contain random coils (Table 2 under supplementary material). The Staphylococcus GUS contains slightly more coils than the $E$. coli GUS. Subsequently, the 3D models GUS from E. coli and Staphylococcus was built with human GUS as template using Discovery Studio. The predicted model is validated using Ramachandran plot for disallowed contacts. Structural superposition $(\mathrm{C} \alpha)$ was carried out between $E$. coli and Staphylococcus GUS models and a RMSD of $4.4 \AA$ was observed. 


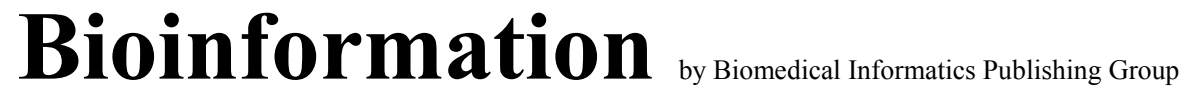

open access

\author{
www.bioinformation.net
}

Hypothesis

The increased random coils in Staphylococcus GUS are proposed to have effects on higher rates of catalysis. The studies of Agarwal [16] elucidate the importance of coiled regions in the process of catalysis. Thus, random coils and loop regions account for greater structural flexibility enzyme towards variation dynamics in solution. Solvent surrounding the enzyme play a role in the enzyme reaction. This provides energy for crossing the activation energy barrier. However, in other cases the required energy is provided by the thermodynamical fluctuations of the solvent. The fluctuations in the hydration shell and bulk-solvent surrounding the enzyme are correlated with the internal protein motions. The side-chains of several surface residues extend into the solvent and the motion of these residues is coupled to the motion of surrounding solvent molecules. Thus structural features aid in the internal motions at fast time-scales to control the chemical environment of the active-site favoring the catalytic step to proceed to the product state. Hence, dynamical coupling allows the transfer of energy from the solvent to the surface regions of the enzyme. This energy transfer changes the behavior of reaction trajectories through the network of protein vibrations that is eventually transferred to the activesite to promote catalysis.

\section{Conclusion:}

Staphylococcus GUS with increased coiling contains all the three catalytic site residues 396Glu, 508Glu and 471Tyr in its loop regions. Moreover, the catalytic residues $396 \mathrm{Glu}$ and 471Tyr were found to have greater solvent accessible surface area compared to the corresponding residues in E. coli GUS. The presence of greater number of charged residues Staphylococcus GUS compared to E. coli GUS is an additional feature for explaining functional difference between them. These differential features provide insights to differentiate the observed catalysis rate in GUS from Staphylococcus and E. coli.

\section{Acknowledgement}

This work is supported by Biotechnology Information System (BTIS) of the Department of Biotechnology, Government of India.

\section{References:}

[01] R. A. Jefferson et al., Proc. Natl. Acad. Sci. USA, 83: 8447 (1986) [PMID: 3534890]

[02] S. Jain et al., Nat. Struct. Biol., 3: 375 (1996) [PMID: 8599764]

[03] R. A. Jefferson, Pl. Mol. Biol. Rep., 5: 387 (1987)

[04] R. A. Jefferson et al., US patent, 6: 391 (2003)

[05] W. Broothaerts et al., Nature, 433: 629 (2005) [PMID : 15703747]

[06] http//ncbi.nlm.nih.gov

[07] http://www.ebi.ac.uk/emboss/align

[08] http://www.ebi.ac.uk/clustalw

[09] http://www.expasy.ch/tools/protparam.html

[10] http://npsa-pbil.ibcp.fr/cgibin/npsa_automat.pl?page=npsa_gor4.html

[11] http://www.rcsb.org/pdb

[12] http://cl.sdsc.edu/ce/ce_align.html

[13] http://swift.cmbi.ru.nl/servers/html/index.html

[14] M. R. Islam et al., J. Biol. Chem., 274: 23451 (1999) [PMID: 10438523]

[15] E. Gasteiger et al., The Proteomics Protocols Handbook, Humana press Inc., Totowa, NJ, 571 (2005)

[16] P. K. Agarwal, Microbial Cell Factories, 5: 2 (2006) [PMID: 16409630]

Edited by P. Kangueane

Citation: Arul et al., Bioinformation 2(8): 339-343 (2008)

License statement: This is an open-access article, which permits unrestricted use, distribution, and reproduction in any medium, for non-commercial purposes, provided the original author and source are credited. 


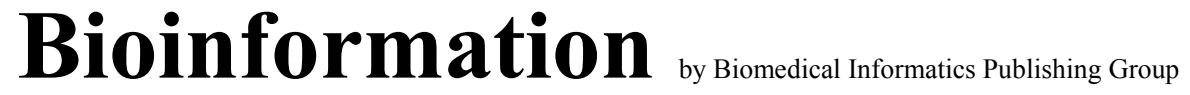

www.bioinformation.net

Hypothesis

\section{Supplementary material}

\begin{tabular}{lll}
\hline \multicolumn{1}{c}{ Properties } & \multicolumn{1}{c}{ E. coli GUS } & \multicolumn{1}{c}{ Staphylococcus sp. GUS } \\
\hline Number of amino acids & 603 & 602 \\
Molecular weight (Dalton) & 68447.0 & 68701.3 \\
Theoretical pI & 5.24 & 5.07 \\
Total number of negatively charged residues (Asp + Glu) & 80 & 90 \\
Total number of positively charged residues (Arg + Lys) & 56 & 62 \\
Molecular formula & $\mathrm{C}_{3068} \mathrm{H}_{4646} \mathrm{~N}_{834} \mathrm{O}_{908} \mathrm{~S}_{22}$ & $\mathrm{C}_{3118} \mathrm{H}_{4720} \mathrm{~N}_{812} \mathrm{O}_{921} \mathrm{~S}_{12}$ \\
Total number of atoms & 9478 & 9583 \\
Computed instability index & 26.68 & 26.54 \\
Aliphatic index & 77.74 & 80.53 \\
\hline
\end{tabular}

Table 1: Predicted physiochemical properties of $\beta$-glucuronidases.

\begin{tabular}{llll}
\hline \multicolumn{1}{c}{ Organism } & Alpha helix (\%) & Beta strand (\%) & Random coil (\%) \\
\hline Escherichia coli & 21.72 & 31.34 & 46.93 \\
Staphylococcus sp. & 22.26 & 28.24 & 49.50 \\
\hline
\end{tabular}

Table 2: The composition of secondary structural elements in $\beta$-glucuronidases from E. coli and Staphylococcus is given. 